\title{
Evidências de validade do inventário de percepção de barreiras atitudinais no trabalho por pessoas com deficiência
}

Gleice Noronha Dias. Prefeitura Municipal de São João del Rei.

Maria Nivalda de Carvalho-Freitas. Universidade Federal de São João del Rei.

Raissa Pedrosa Gomes Tette. Universidade Federal de São João del Rei.

\section{Resumo}

O objetivo deste estudo é relatar o processo de construção e testes iniciais para identificar evidências de validade do Inventário de Percepção de Barreiras Atitudinais no Trabalho por pessoas com deficiência (IBAT-PD). Realizaram-se dois estudos: (1) a construção do inventário, a avaliação de adequação da representação do construto por meio da análise fatorial e a análise da consistência interna; (2) avaliação de evidências de validade convergente do IBAT-PD com o construto de socialização organizacional. No primeiro estudo foi realizado um survey com 54 pessoas com deficiência de várias regiões do país. No segundo estudo, foi realizada uma investigação com 17 funcionários com deficiência de sete prefeituras municipais mineiras. A análise fatorial indicou uma solução unifatorial para o instrumento. Os resultados demonstraram correlações de forte magnitude entre os construtos, indicando que, apesar de serem necessários estudos com amostras maiores, o IBAT-PD tem características que referendam sua utilização em situações de trabalho.

Palavras-chave: deficiência; socialização; barreiras; inclusão.

\begin{abstract}
Evidence of validity of the inventory of perceptions of attitudinal barriers at work by people with disabilities. The objective of this study is to report the construction process and initial tests to identify evidence of validity of the Inventory of Perceptions of Attitudinal Barriers at Work by people with disabilities (IABW-PWD). Two studies were carried out: (1) construction of the inventory and the evaluation of the adequacy of the representation of the construct through factorial analysis and internal consistency analysis; (2) evaluation of evidence of convergent validity of IABT-PWD with the construct of organizational socialization. In the first study, a survey was conducted with 54 people with disabilities from various regions of the country. In the second study, an investigation was carried out with 17 employees with disabilities from seven municipalities in Minas Gerais. The factorial analysis indicated a unifactorial solution and evidence of reliability of the instrument. The results showed strong correlations between organizational socialization and IABW-PWD and useful characteristics to understand work situations of people with disabilites.
\end{abstract} Keywords: disability; socialization; barriers; inclusion.

\section{Resumen}

Evidencias de validez del inventario de percepción de barreras actitudinales en el trabajo por las personas con discapacidad. El objetivo de este estudio es relatar el proceso de construcción y pruebas iniciales de evidencias de validez del Inventario de Percepción de Barreras Actitudinales en el Trabajo por personas con discapacidad (IBAT-PD). Se realizaron dos estudios: (1) la construcción del inventario y la evaluación de la idoneidad construyen la representación por medio de análisis y análisis de la consistencia interna del factor; (2) evaluación de evidencias de validez convergente del IBAT-PD con el constructo de socialización organizacional. En el primer estudio se realizó un survey con 54 personas con discapacidad de varias regiones del país. En el segundo estudio, se realizó una investigación con 17 funcionarios con discapacidad de siete prefecturas municipales mineras. El análisis factorial indicó una solución unifactorial para el instrumento. Los resultados demostraron correlaciones de fuerte magnitud entre los constructos, indicando que, a pesar de ser necesarios otros estudios con muestras mayores, el IBAT-PD tiene características que refrendan su utilización en situaciones de trabajo.

Palabras clave: deficiencia; socialización; barreras; inclusión 
A relevância dos estudos acerca de pessoas com deficiência pode ser corroborada por meio dos dados do Instituto Brasileiro de Geografia e Estatística (IBGE) em censo realizado em 2010: o Brasil possui cerca de 45,6 milhões de pessoas com algum tipo de deficiência, representando aproximadamente $24 \%$ da população. Entretanto, os dados da Relação Anual de Informações Sociais (RAIS) indicaram que existem 381,3 mil vínculos empregatícios declarados como sendo de pessoas com deficiência, representando somente $0,77 \%$ do total dos vínculos empregatícios do país (Ministério do Trabalho e Emprego [MET], 2014).

Como resultado do esforço de inserir as pessoas com deficiência no trabalho surgiram as legislações, instituindo políticas públicas com reserva de vagas para essas pessoas. A obrigatoriedade em inserir as pessoas com deficiência no trabalho traz novas questões para as organizações, que são a convivência com pessoas com deficiência e a gestão da diversidade. Tais organizações se veem impactadas pela necessidade de adequação das condições e práticas do trabalho visando à adaptação desse público (Carvalho-Freitas, 2007).

Pesquisas demonstram que as desvantagens intelectuais, físicas ou sensoriais enfrentadas pelas pessoas com deficiência podem ser maiores ou menores em vista das contingências históricas, sociais e espaciais nas quais essas pessoas fazem parte (Carvalho-Freitas, 2007; Roulstone, 2012). Considerando que a vida de uma pessoa com deficiência está frequentemente associada a desvantagens que se materializam por intermédio de barreiras que dificultam seu acesso aos diversos espaços na sociedade, depreende-se que a forma como está organizada nossa sociedade contribui para o processo de exclusão e preconceito que permeia a vida dessas pessoas.

As barreiras, segundo a Lei 13.146 de 6 de julho de 2015, são quaisquer entraves ou obstáculos que dificultam o acesso, a liberdade de movimento e a circulação com segurança das pessoas com deficiência. Sassaki (2010) discute que para a promoção da acessibilidade é necessário eliminar da sociedade todas as barreiras que impedem o acesso e o desenvolvimento profissional, escolar, social e cultural das pessoas com deficiência. $\mathrm{O}$ autor diferenciou seis dimensões de acessibilidade que contemplam as barreiras que fazem parte do cotidiano de pessoas com deficiência: arquitetônicas (obstáculos que impedem as pessoas de desfrutarem e ocuparem o espaço físico), comunicacionais (presentes quando as informações não estão disponíveis para todos, seja porque não existem ou porque não são apresentadas de forma acessível), metodológicas (padronização das atividades nas organizações de trabalho e ausência de adequações nos postos de trabalho), instrumentais (falta de recursos de Tecnologia Assistiva), programáticas (referentes ao conjunto de dificuldades derivadas das políticas e normas organizacionais) e atitudinais (tendência desfavorável na forma de se sentir e se comportar em relação às pessoas com deficiência). Embora se reconheça que todas essas barreiras necessitem ser eliminadas para garantir o acesso e permanência de pessoas com deficiência no trabalho, entende-se que da barreira atitudinal derivam-se todas as demais barreiras, pois serão as pessoas com atitudes favoráveis ou desfavoráveis em relação ao trabalho das pessoas com deficiência que definirão a forma como as questões arquitetônicas, comunicacionais, metodológicas, instrumentais ou programáticas serão realizadas. Por esse motivo, a questão das barreiras atitudinais foi o foco principal da presente investigação.

Por atitude entende-se a "tendência persistente de se sentir e se comportar de maneira favorável ou desfavorável em relação a uma determinada pessoa, um objeto ou uma ideia" (Hitt, Miller, \& Colella, 2007, p. 142), sendo o preconceito definido como uma atitude negativa injusta (Hitt et al., 2007). A atitude contém elementos cognitivos, que se referem às crenças construídas previamente; afetivos, que se referem aos sentimentos que se tem em relação ao objeto, pessoa ou ideia; e elementos comportamentais referentes à intenção do indivíduo de agir de determinada maneira em relação àquela questão específica, quer seja um objeto, pessoa ou ideia (Ajzen, 1991; Fishbein \& Ajzen, 1975).

Nesse sentido, a barreira atitudinal pode ser entendida como um comportamento desfavorável ou preconceito que se tem em relação às pessoas com deficiência e que, na maioria das vezes, leva a uma discriminação, entendida, conforme Hitt et al. (2007), como o comportamento que se materializa em um tratamento desigual para com esses indivíduos. No caso das pessoas com deficiência a discriminação está associada ao conceito de estigma que, numa primeira acepção, se refere a "sinais corporais com os quais se procurava evidenciar alguma coisa de extraordinário ou mau sobre o status moral de quem os apresentava" (Goffman, 2008, p. 11). Assim, pode-se considerar o estigma como um critério de diferenciação e distinção utilizado em relação às pessoas com deficiência. Está referido, assim, 
a um atributo (deficiência), mas se configura em uma relação que distingue e atribui características de desvalorização aos portadores desse atributo, discriminando-os por meio de tratamento desigual.

As barreiras atitudinais, focalizadas nesta pesquisa, estão incrustadas no seio da sociedade atual, estando presentes quando as atitudes das pessoas influenciam negativamente a vida de pessoas com deficiência. Na medida em que a deficiência leva o indivíduo a se sentir incapaz de atender às expectativas dos outros, podem ocorrer efeitos negativos sobre os seus valores pessoais, resultando em retraimento, insegurança e depressão (Stone-Romero, Stone, \& Lukaszewski 2006). Esses sentimentos vão resultar numa ameaça ao desempenho das pessoas com deficiência, pois as colocam numa situação de vulnerabilidade que pode afetar sua integração nas atividades laborais.

Considerando que várias pesquisas identificaram as dificuldades das pessoas com deficiência para se inserirem no mercado de trabalho e que um processo cuidadoso de adaptação da pessoa com deficiência numa organização será preponderante para que ela se mantenha no trabalho (Carvalho-Freitas, Marques, \& Scherer, 2004; Neri, Pinto, Soares, \& Costilha, 2003; Shakespeare, 2012; Stone-Romero et al., 2006; Tanaka \& Manzini, 2005; dentre outros), reforça-se a necessidade de inserção dessas pessoas no ambiente laboral, mas de uma forma que viabilize a sua permanência mediante a análise de fatores que favoreçam o seu desempenho. Para que se apresente uma inserção efetiva é necessária a preparação de todos os envolvidos nos aspectos profissionais e sociais relacionados ao trabalho, para garantir a melhor adaptação dessas pessoas no contexto organizacional.

Nesse sentido, o estudo da temática da socialização organizacional é determinante para se entender a inclusão de pessoas com deficiência no trabaIho. Isso porque a socialização organizacional se refere ao processo de adaptação e integração das pessoas às organizações sendo, de acordo com Borges e Albuquerque (2004), "a maneira de um indivíduo tornar-se membro de um grupo, organização ou da sociedade, entendendo que o indivíduo socializado é o sujeito ou personagem principal desse processo" (p.332).

Em procedimentos de adaptação, além dos requisitos técnicos necessários ao cargo, o indivíduo irá compartilhar os saberes sobre os valores, cultura, história passada e potencial dos membros dos grupos pertencentes à organização. Caso a socialização ocorra de forma desfavorável, trará efeitos negativos para todos os atores envolvidos e para a instituição (Wagner \& Holenbeck, 2006). Este processo, que têm como objetivo tornar o indivíduo um membro da organização (Borges \& Albuquerque, 2004), pode estar mais relacionado com um conjunto de ações denominadas 'táticas individuais' (Griffin, Colella, \& Goparaju, 2000) ou, num outro extremo, designadas 'táticas organizacionais' (Van Maanen \& Schein, 1979). As principais táticas individuais consistem em busca de informação e feedback; construção de relacionamentos, negociação de mudança nas atividades, autoavaliação, autogerenciamento das informações, envolvimento em atividades relacionados ao trabalho e observação (Griffin et al., 2000). Já as táticas organizacionais referem-se às ações realizadas pelos membros da organização a fim de ensinar ao novo colaborador os comportamentos e habilidades que são desejáveis e habituais naquele contexto de trabalho. Estas ações nem sempre são planejadas e funcionam também como elemento de fixação e manutenção da cultura organizacional.

Destaca-se, dentre os estudos nessa temática, a investigação de Borges, Silva, Melo e Oliveira (2010) que validou um inventário de socialização organizacional, a partir de ampla revisão da literatura sobre o tema, que permite identificar, a partir do próprio olhar do sujeito, seu nível de socialização organizacional. Essa validação permitiu identificar sete fatores que indicam o nível de socialização do trabalhador: 1. Competência e Proatividade: demonstra o domínio do participante em relação às atividades que executa no trabalho e os próprios esforços empreendidos em favor de sua integração à organização. 2. Qualificação Profissional: percepção que o indivíduo tem sobre a sua preparação para o cargo (conhecimentos, informações e experiência). 3. Linguagem e Tradição: percepção que o indivíduo tem sobre seu desempenho em utilizar os termos técnicos e linguagem condizente com a cultura da organização e o quanto o indivíduo percebe a identidade da organização, através do conhecimento adquirido da sua história e tradições. 4. Integração com as pessoas: a perspectiva que o indivíduo possui em ser incluído no grupo, se sentindo aceito e apoiado pelos demais. 5. Objetivos e Valores Organizacionais: o quanto o indivíduo conseguiu apreender os valores da organização (metas, objetivos, prioridades), se identificando com eles. 6. Acesso às Informações (Políticas): o quanto o indivíduo se percebe conhecendo a estrutura e funcionamento da organização. 7. Não-Integração 
à Organização: indica a ausência do conhecimento sobre os processos organizacionais e da cultura organizacional, e a ausência de domínio da linguagem e do trabalho.

Tendo esse cenário por referência, a presente pesquisa objetiva contribuir com o avanço das discussões e do conhecimento junto aos estudos sobre socialização organizacional de pessoas com deficiência realizados no Brasil (Assis, 2012; Carvalho-Freitas, Toledo, Nepomuceno, Suzano, \& Almeida, 2010; Moreira, Cappelle, Miranda, \& Onuma, 2011), sobretudo considerando os preconceitos e discriminações sob a forma de barreiras atitudinais, que desfavorecem esse processo de adaptação. Dessa forma, esse artigo tem por objetivo relatar o processo de construção e testes iniciais para identificar evidências de validade do Inventário de Percepção de Barreiras Atitudinais no Trabalho por pessoas com deficiência (IBAT-PD), por meio de dois estudos com amostras diferentes. O primeiro visou construir e buscar evidências de validade para um instrumento de barreiras atitudinais, e o segundo visou ampliar a análise da validade do construto proposto por meio de validação convergente, verificando se existe relação entre barreiras atitudinais e a socialização organizacional na perspectiva das próprias pessoas com deficiência. A hipótese é de que existe correlação de forte magnitude entre os construtos de barreiras atitudinais e de socialização organizacional.

\section{Método}

Visou-se, no primeiro momento, a construção e validação do Inventário de Percepção de Barreiras Atitudinais no Trabalho por parte de Pessoas com Deficiência (IBAT-PD) e na sequência, apresentar os resultados de sua validação convergente com o construto de socialização organizacional.

\section{Participantes}

Os critérios definidos para a participação no primeiro estudo foram possuir deficiência e ter vínculo de trabalho no momento do estudo. O questionário foi divulgado por meio de uma rede social em 15 grupos específicos de pessoas com deficiência, sendo utilizado o método eletrônico de survey de desenho transversal pela internet. Dentre os 54 participantes que responderam à pesquisa, houve um equilíbrio na distribuição entre os dois sexos, sendo 51,85\% masculino e 48,15\% feminino, com média de idade de 36,6 anos. No nível de escolaridade, houve predominância de pessoas com nível superior (53,7\%). A menor incidência de participação foi de pessoas com nível de mestrado (5,55\%).

Essas pessoas com deficiência são provenientes de quatro regiões do Brasil e 13 estados diferentes do país, sendo a maior representatividade do Sudeste (70,34\%), sobretudo de Minas Gerais (40,74\%), seguido de São Paulo (18,52\%). A pesquisa não obteve respondentes apenas da Região Norte do Brasil. Entre os participantes, $53,70 \%$ eram solteiros, $22,22 \%$ casados e $12,96 \%$ divorciados. As outras possibilidades (união estável, viúvo, desquitado e outros) somaram 11,1\%.

Em relação ao tipo de deficiência, a frequência é de $77,77 \%$ de pessoas com deficiência física, demonstrando a predominância desse tipo de deficiência entre os participantes. Apresentando outros tipos de deficiência, 11,11\% de pessoas possuíam deficiência visual, 5,55\% apresentaram deficiência auditiva e 5,55\% de pessoas reabilitadas de acidentes de trabalho.

O nível salarial com maior frequência entre os participantes foi de dois a três salários mínimos, em $37,03 \%$ dos casos. O nível de um salário mínimo também obteve frequência bastante alta, de 33,33\%; por outro lado, salário superior a sete salários mínimos alcançou a menor frequência entre eles, 9,25\%.

O segundo estudo contemplou 17 pessoas com deficiência, funcionárias de prefeituras de sete municípios de diferentes regiões do Campo das Vertentes, em Minas Gerais. As instituições pesquisadas, prefeituras, têm a obrigatoriedade de realizar concursos públicos para o provimento dos cargos para pessoas com deficiência em sua estrutura, ancorados pela Lei 8.112. Por conseguinte, a principal forma de admissão das pessoas pesquisadas foi mediante a reserva de vagas para pessoas com deficiência em concursos dessas prefeituras.

Nesse sentido, 70,58\% dos participantes são servidores efetivos que alçaram o emprego por meio da Lei de Cotas e $23,52 \%$ (três pessoas com deficiência) foram contratados pelos gestores, independente da lei. Contudo, dois deles passaram a ser servidores efetivos e constam entre os servidores cotistas da instituição.

Os participantes distribuíram-se da seguinte forma: $70,58 \%$ dos respondentes são do sexo masculino e $29,41 \%$ do sexo feminino. Esses dados são próximos ao que demonstra a RAIS (MTE, 2014), cujos dados revelam $64,45 \%$ de representação masculina de pessoas com deficiência em empregos formais no Brasil. Em relação à faixa etária, predominou a participação de pessoas com deficiência com idade superior 
a 40 anos, representando $58,81 \%$ dos participantes. Quanto à escolaridade, observa-se uma taxa maior de pessoas com deficiência com o Ensino Médio completo (35,29\%). Há apenas uma pessoa com a Graduação completa e não houve, entre os participantes da pesquisa, nenhuma pessoa com deficiência com escolaridade em níveis de pós-graduação. As informações concernentes ao estado civil delas revelaram que $41,17 \%$ são solteiras, $29,41 \%$ casadas, $17,64 \%$ com união estável e 11,76\% são divorciados.

Entre as pessoas com deficiência das prefeituras, há um predomínio da deficiência física, com $76,47 \%$ dos respondentes da pesquisa. Em números absolutos, dos 17 participantes, 13 relataram deficiência física, três relataram deficiência visual e um relatou deficiência auditiva.

A maior parte dos respondentes exerce atividades operacionais dentro da prefeitura $(82,32 \%)$ e o nível salarial predomina em $64,70 \%$ na faixa de um a dois salários mínimos, 35,29\% recebem como remuneração dois a três salários mínimos e nenhum participante recebe remuneração superior a três salários mínimos.

O tempo de trabalho na prefeitura também é fator de análise importante, pois 58,82\% dos respondentes trabalham há mais de cinco anos nas instituições pesquisadas, apenas $11,76 \%$ das pessoas com deficiência trabalham por período menor que um ano e $23,52 \%$ trabalham pelo período de três a quatro anos.

\section{Instrumentos}

A construção do IBAT - PD realizada no primeiro estudo foi pautada na revisão dos estudos de Sassaki (2003), dos estudos sobre atitudes de Fishbein e Ajzen (1975) e sobre preconceito e estigma (Goffman, 2008; Stone-Romero et al, 2006; Roulstone, 2012). O instrumento pretende investigar as barreiras atitudinais percebidas pelas pessoas com deficiência no contexto do trabalho.

Os dez itens foram elaborados de forma que fossem consideradas as percepções das pessoas com deficiência em relação às atitudes de colegas de trabaIho, chefias-imediatas, indivíduos de outros setores da instituição, bem como a postura geral do empregador em relação a elas, externando atitudes como preconceito (avaliação negativa), impedimento de acesso às atividades, supervalorização, superproteção, comparações e descrédito, tendo por referência as contribuições de Sassaki (2003), Stone-Romero et al (2006), Roulstone (2012) e Goffman (2008). Os itens do Inventário de
Percepção de Barreiras Atitudinais no Trabalho por parte das Pessoas com Deficiência - IBAT-PD foi submetido a um painel de especialistas. Participaram do painel: três professores-pesquisadores da área relacionada à inclusão de pessoas com deficiência no trabalho e seis mestrandos com afinidade na temática. Os juízes examinaram e sugeriram alterações visando realizar a validade de construto, de acordo com as recomendações de Pasquali (2009). Na etapa seguinte, o inventário foi aplicado em uma amostra pré-teste no sentido de se realizar a análise semântica. A amostra foi composta por 19 estudantes com deficiência, os quais sugeriram poucas modificações nos itens. Por fim, o questionário ficou composto por 10 itens, com questões fechadas, dispostas em escala Likert, de escolha forçada, de seis pontos (1 - discordo totalmente até 6 - concordo totalmente).

Já no segundo estudo, foram aplicados os seguintes instrumentos para a coleta de dados: o Inventário de Socialização Organizacional (ISO) desenvolvido por Borges et al. (2010), o Inventário de Percepção de Barreiras Atitudinais no Trabalho por parte de Pessoas com Deficiência (IBAT-PD), construído e validado para a presente pesquisa, e um questionário de dados sociodemográficos das pessoas com deficiência, com o objetivo de construir um perfil dos participantes e de se verificar se a proporção das respostas nos inventários dependiam de fatores sociodemográficos. O Inventário de Socialização Organizacional (ISO) foi aplicado em todos os participantes da pesquisa. Ele é constituído de 45 itens, em escala Likert, com as possibilidades de escolha que vão de -2 (forte discordância) a +2 (forte concordância). Todas as questões do ISO são formuladas na forma negativa. O IBAT-PD também foi aplicado em todos os participantes da pesquisa.

\section{Procedimentos de Coleta de Dados}

A pesquisa foi aprovada pelo Comitê de Ética e para o primeiro estudo foram aplicados os procedimentos de análise da adequação da representação do construto, visando identificar evidências de validade, por meio da análise fatorial e da análise de consistência interna (Pasquali, 2009). Todas as respostas dos 54 participantes do processo de validação foram incluídas na pesquisa. Verificou-se que não ocorreram casos faltosos (missing values). Os 10 itens do inventário também foram mantidos, pois apresentaram carga fatorial superior a 0,30 e nenhuma ambiguidade. 


\section{Procedimentos de Análise de Dados}

Os dados foram submetidos ao teste KomogorovSmirnov e Shapiro-Wilk. Avaliou-se que atendiam aos pressupostos de normalidade, pois todos os dados tiveram nível de significância menor que 0,05. Já o índice de Kaiser-Meyer-Olkin (KMO =0,848) e o teste de esfericidade de Bartlett (significativo a $p<0,001$ ) apontaram a fatorabilidade dos dados. A amostra obtida para a realização da análise fatorial do IBAT-PD foi de cinco respondentes para cada variável, conforme orientações de Hair, Anderson, Tatham e Black (2005). Os dados foram submetidos à análise dos componentes principais por meio da rotação ortogonal varimax com o objetivo de determinar o número mínimo de fatores que respondessem pela máxima variância dos dados, maximizando as altas correlações e minimizando as baixas (Tabachnick \& Fidell, 2007). Além disso, foram analisadas possíveis relações de dependência entre os resultados do inventário e os dados sociodemográficos, tendo sido utilizadas as seguintes técnicas estatísticas: estatísticas descritivas e teste Qui-Quadrado de Independência.
No segundo estudo, foram verificadas as correlações entre o IBAT-PD e o ISO, por meio da Correlação de Spearman, visando identificar se a hipótese de relação entre percepção de barreiras atitudinais e percepção de socialização organizacional se confirmava empiricamente. Também foram analisadas possíveis relações de dependência entre os resultados do inventário e os dados sociodemográficos, tendo sido utilizadas estatísticas descritivas e teste Qui-Quadrado de Independência.

\section{Resultados}

Os dados serão apresentados em duas fases, sendo a primeira fase referente aos índices de validação do IBAT-PD, realizada mediante análise fatorial exploratória com a primeira amostra da pesquisa. A segunda análise trata-se de uma validação convergente entre os construtos de barreiras atitudinais e o de socialização organizacional, realizada com a segunda amostra da pesquisa.

Tabela 1. Matriz Fatorial dos Itens do IBAT-PD

O preconceito (avaliação negativa) exercido pela chefia imediata das pessoas com deficiência.

O preconceito (avaliação negativa) exercido por servidores/funcionários de outros setores da Instituição. 


\section{Primeiro Estudo: Evidências de Validade do IBAT-PD}

A análise estatística inicial do IBAT-PD apontou como resultado a divisão dos itens em dois fatores. Esses fatores apresentaram eigenvalues superiores a 1,0 explicando $71,68 \%$ da variância total. Avaliando as cargas, comunalidades e pressuposto teórico adotado, o mesmo procedimento foi refeito visando avaliar a adequação de uma solução unifatorial para o inventário, pois os resultados dos dois fatores, embora empiricamente distintos, apresentavam sobreposições conceituais, indicando a organização do inventário em um único fator como o procedimento mais adequado a ser adotado. Os valores obtidos, nessa segunda análise, foram: Kaiser-Meyer-Okin (KMO =0,848) e Barlett ( $p<0,001$ ), explicando $56 \%$ da variância total. Foram mantidos os 10 itens iniciais, pois todos tiveram carga fatorial superior a 0,30 na solução de um único fator. Para verificar a confiabilidade do fator, foi utilizado o coeficiente alfa de Cronbach (Tabachnick \& Fidell, 2007), obtendo o valor de 0,914. A Tabela 1 apresenta a matriz fatorial dos itens do Inventário de Percepção de Barreiras Atitudinais no Trabalho por parte das Pessoas com Deficiência - IBAT-PD, além das cargas dos itens do fator, o eigenvalue, o percentual de variância explicada e a consistência interna, representando bons resultados em estudo exploratório (Pasquali, 1999).

Tendo a solução fatorial de um único fator, pode-se inferir como característica geral do instrumento que as barreiras atitudinais percebidas pelas pessoas com deficiência em situações de trabalho se referem à percepção de serem comparadas com as demais pessoas, à inclinação das pessoas em desacreditar no desempenho delas, além de qualificarem desfavoravelmente as ações delas. Pode-se perceber uma maior frequência de escolha das opções intermediárias, com tendência a discordar pouco das afirmativas do questionário (Média $=3,03$ e Desvio Padrão $=1,39$ ). Além disso, não foi identificada nenhuma relação de dependência (teste qui-quadrado) entre o IBAT-SP e os dados sociodemográficos.

\section{Segundo Estudo: Validação Convergente}

A análise de correlação entre o IBAT-PD e o ISO foi realizada por meio do coeficiente de correlação de Spearman e os resultados alcançados podem ser observados na Tabela 2. A opção pelo coeficiente de correlação de Spearman foi devido ao fato da amostra não obedecer a uma distribuição normal. Nesse caso, foram utilizados testes não-paramétricos. Para a avaliação da magnitude das correlações, foi adotada a seguinte classificação, segundo Hulley, et al., (2003): coeficientes de correlação < 0,4 (correlação de fraca magnitude), $>0,4$ a $<0,5$ (de moderada magnitude) e > 0,5 (de forte magnitude). A Tabela 2 demonstra a existência de correlações estatisticamente significativas ( $p<0,05$ e $p<0,01$ ) entre os construtos de percepção de barreiras atitudinais e o de socialização organizacional, o que indica que os resultados encontrados não são decorrentes de erro amostral. Verificou-se também que as correlações existentes são todas de forte magnitude.

Tabela 2. Valores de Média, Desvio-Padrão e Correlação de Spearman entre os Fatores de Percepção de Barreiras Atitudinais de Pessoas com Deficiência (IBAT-PD) e os Fatores de Socialização Organizacional (ISO)

IBAT-PD

Fator

$(M=2,86 / D P=1,12)$
Competência e Proatividade $(M=4,68 / D P=0,41)$
Integração às Pessoas $(M=3,79 / D P=0,92)$
$-0,79^{* *}$
Não-integração à Organização $(M=1,94 / D P=1,03)$
$0,58^{*}$
Acesso às Informações (Políticas) ( $M=4,13 / D P=0,77)$
Qualificação Profissional $(M=4,47 / D P=0,87)$
Objetivos e Valores Organizacionais ( $M=4,31 / D P=0,81)$
$-0,64^{* *}$
Linguagem e Tradição $(M=3,73 / D P=0,70)$
$-0,56^{*}$

Notas: ${ }^{*} p<0,05 ;{ }^{* *} p<0,01$

Fonte: Dados da pesquisa. 
Os resultados indicam que: (1) quanto mais as pessoas com deficiência se sentem integradas às outras pessoas, sendo incluídas no grupo, sentindo-se participativas e apoiadas pelos membros da organização, menos percebem barreiras atitudinais no ambiente de trabalho; (2) quanto mais as pessoas com deficiência se sentem não-integradas à organização, isto é, não conhecendo os processos e a cultura organizacional e não dominando a linguagem do trabalho, mais elas percebem as barreiras atitudinais dentro da organização; (3) quanto mais elas conhecem os objetivos e valores organizacionais, isto é, quanto mais elas sabem identificar as metas, objetivos e prioridades organizacionais, menos elas percebem as barreiras atitudinais na organização; (4) quanto mais elas se apropriam da linguagem e tradição da organização, menos elas percebem as barreiras atitudinais.

Em relação às barreiras atitudinais verifica-se que os participantes têm uma tendência a discordar pouco em relação às afirmativas relacionadas às atitudes negativas em situações de trabalho (Média $=2,86 \mathrm{e}$ Desvio-Padrão $=1,12$ ).

Considerando os fatores do ISO, o fator com uma maior tendência de concordância foi

Competência e Proatividade, que analisa o quanto a pessoa é produtiva, eficaz e se se antecipa na busca de informações para a melhoria do desempenho (Média $=4,68$ e Desvio-Padrão $=0,41$ ). O fator Qualificação Profissional corresponde ao domínio das tarefas advindas de uma experiência profissional e de se ter uma preparação para o exercício do cargo (Média $=4,47$ e Desvio-Padrão = 0,87). Já a definição do fator Objetivos e Valores Organizacionais demonstra que a pessoa está informada sobre as metas e as prioridades da organização e a identificação do trabalhador com os valores da instituição (Média $=4,31$ e Desvio-Padrão $=0,81$ ). Portanto, percebe-se que estes dois fatores também tiveram tendência a uma concordância dos participantes da pesquisa.

Quanto ao fator Acesso às Informações (Políticas), as pessoas com deficiência também tiveram resultados próximos aos dois fatores anteriores (Média $=4,13 \mathrm{e}$ Desvio-Padrão $=0,77$. Este fator condiz com o fato de a pessoa conhecer sobre os processos organizacionais, as datas importantes e as relações de poder dentro da organização. O fator Linguagem e Tradição corresponde ao pleno conhecimento da linguagem utilizada pelas pessoas e pela organização (Média $=3,73$ e DesvioPadrão $=0,70$ ), demonstrando alguma incerteza. Já o fator denominado Integração às Pessoas visa a mensurar o sentimento das pessoas quanto à sua aceitação no grupo e participação na tomada de decisões (Média = 3,79 e Desvio-Padrão = 0,92). O fator Não-integração à Organização foi o que obteve menor resultado (Média $=1,94$ e Desvio-Padrão $=1,03$ ). No entanto, todas as questões foram formuladas na sua forma negativa. Assim, o menor valor neste fator corresponde ao fato de que as pessoas se sentem bem integradas à organização. Mediante os resultados apresentados pelo ISO, em geral, os sujeitos pesquisados apresentam bons resultados nos escores que demonstram uma boa percepção de estarem socializados nas instituições onde trabaIham. Também nesse estudo, não foram identificadas nenhuma relação de dependência (teste qui-quadrado) entre o IBAT-SP e os dados sociodemográficos.

\section{Discussão}

A partir dos resultados obtidos no desenvolvimento do Inventário de Percepção de Barreiras Atitudinais no Trabalho por parte das Pessoas com Deficiência (IBAT- PD), foi identificado, empiricamente, que ele apresenta evidências de validade de conteúdo e fidedignidade, além de ser capaz de se correlacionar significativamente com outro construto, no presente caso, a socialização, o que indica sua validade convergente e consolida bom potencial para avaliação de barreiras atitudinais em situações de trabalho. Embora os arranjos exploratórios da análise fatorial indicassem a existência de dois fatores explicando expressiva variância, a análise dos itens indicava uma sobreposição conceitual de difícil solução teórica. A solução de um único fator para o inventário permitiu uma coerência e consistência interna significativa entre os itens, indicando que os itens medem o mesmo construto de barreiras atitudinais.

Como a proposta do inventário de barreiras atitudinais se destinava a pessoas com deficiências, escolaridades e funções diversas, os resultados permitiram identificar que, em princípio, a percepção de barreiras atitudinais independe desses fatores. Essa relação de independência foi identificada nos dois estudos realizados. No entanto, novas pesquisas são necessárias para identificar principalmente se a percepção de barreiras atitudinais é realmente independente do tipo de deficiência, pois pesquisas anteriores têm mostrado que os gestores avaliam diferentemente o desempenho das pessoas, conforme o tipo de deficiência, o que pode 
fazer com que suas atitudes variem conforme o tipo de deficiência que a pessoa possua (Suzano, CarvalhoFreitas, Tette, Brighenti, \& Vieira-Silva, 2014).

Também os resultados de percepção das pessoas com deficiência de pouca discordância em relação a atitudes negativas em relação a elas, nos dois estudos, têm sido encontrados em outras pesquisas com pessoas com deficiência no trabalho, demonstrando que o preconceito pode aparecer de formas explícitas ou de formas mais sutis nas relações de trabalho (Leite \& Lorentz, 2008; Neves-Silva, Prais, \& Silveira, 2015; Serrano \& Brunstein, 2007), fazendo com que as pessoas com deficiência, em pesquisas quantitativas, tendam a responder buscando escolhas intermediárias. De qualquer forma, esse tipo de resposta revela a incerteza e a ambiguidade que muitas vezes se revestem as relações das pessoas com deficiência no trabalho.

Por outro lado, as correlações entre a percepção de barreiras atitudinais e fatores de socialização organizacional que se referem à integração, não-integração, conhecimento dos objetivos e valores organizacionais, e apropriação da linguagem e tradição da organização, ajuda na ampliação da compreensão de que a socialização organizacional das pessoas com deficiência está relacionada diretamente com a atitude das pessoas no cotidiano de trabalho. Esse resultado reforça a importância de atitudes favoráveis que evitem a comparação e inferiorização das pessoas em processo de socialização, ratificando o estudo de Guedes (2007), que avaliou as barreiras atitudinais praticadas contra pessoas com deficiência estudantes de instituições de ensino privadas, demonstrando como atitudes de comparação podem ser prejudiciais ao desempenho delas. $O$ autor confirma que essa atitude ressalta não suas possibilidades, e sim suas falhas, faltas e deficiências, colocando-as numa posição de inferioridade em relação aos outros.

Nesse sentido, é importante aliar estratégias de socialização como treinamentos de integração e capacitações e a necessidade de melhorias na difusão de informações e na transmissão da cultura organizacional com uma atitude favorável por parte da organização em relação às possibilidades de trabalho de pessoas com deficiência. Esses resultados reforçam a importância da socialização organizacional e ampliam os achados de Carvalho-Freitas et al (2010) e de Maccali, Kuabara, Roseli e Takahashi (2015), pois além da importância da sistematização das estratégias de socialização, o presente estudo indica a importância da atitude das pessoas para que as pessoas com deficiência efetivamente se sintam adaptadas à organização. Além disso, esses resultados corroboram a importância da receptividade das pessoas para incluir as pessoas com deficiência no grupo, para que se sintam identificadas com a equipe, aceitas e acolhidas e, ainda, participantes das decisões da sua unidade de trabalho. Percebe-se como essencial esse comportamento das pessoas para que as pessoas com deficiência não se sintam impactadas por atitudes preconceituosas, nas quais companheiros de setor e/ou chefias imediatas invalidem seus esforços em relação ao trabalho, comparando seu desempenho e/ou agindo com descrédito sobre as suas possibilidades e potencialidades.

Nesse sentido, considera-se que a percepção de ausência de barreiras atitudinais parece ser preponderante para que as pessoas com deficiência se sintam integradas e compartilhando dos objetivos e valores da instituição onde trabalham. Várias pesquisas demonstram a importância da convivência produtiva com colegas e chefias imediatas como motores do processo de socialização organizacional de pessoas com deficiência (Assis, 2012; Ávila-Vítor, \& Carvalho-Freitas, 2012; Carvalho-Freitas et al., 2010; Guedes, 2007). O levantamento dessas situações poderá fomentar práticas dos gestores e profissionais de Gestão de Pessoas objetivando mudanças nas atitudes dos indivíduos em relação às pessoas com deficiência, pois estas mudanças de comportamento minimizariam os obstáculos enfrentados por elas no cotidiano de trabalho. Além disso, deve se considerar também que o fato do IBAT-PD ser uma medida curta, ele permite a sua aplicação em conjunto com outras escalas de comportamento organizacional e de gestão de pessoas, facilitando a identificação de questões que podem ter implicações práticas para a inclusão de pessoas com deficiência nas equipes de trabalho e nas organizações. Também as barreiras atitudinais, como uma medida que avalia a percepção de pessoas com deficiência, podem ser aplicadas a trabalhadores com deficiência que atuam em diferentes contextos, setores e situações de emprego. A simplicidade do inventário também permite que ele seja aplicado em trabalhadores de baixa escolaridade, situação comum entre pessoas com deficiência no país.

Esse estudo tem como principal limitação o tamanho da amostra nos dois estudos realizados. Novas pesquisas são necessárias para avaliar outras características de validade do instrumento em amostras maiores e em outros contextos. 


\section{Referências}

Ajzen, I. (1991). The theory of planned behavior. Organizational Behavior and Human Decision Processes, 50(2), 179-211. doi: 10.1016/0749-5978(91)90020-T

Assis, A. M. (2012). Socialização de pessoas com deficiência: um estudo de caso numa organização de grande porte (Dissertação de mestrado não publicada). Universidade Federal de São João del-Rei. São João del-Rei, MG, Brasil.

Ávila-Vítor, M. R. C., \& Carvalho-Freitas, M. N. (2012). Valores organizacionais e concepções de deficiência: a percepção de pessoas incluídas. Psicologia: Ciência e Profissão, 32(4), 792-807. doi: 10.1590/S1414-98932012000400003

Borges, L. O., \& Albuquerque, F.J. B. (2004). Socialização Organizacional. In J. C. Zanelli, J. E. Borges-Andrade, \& A. V. B. Bastos (Orgs.), Psicologia, organizações e trabalho no Brasil (pp. 331-356). Porto Alegre: Artmed.

Borges, L. O., Silva, F. H. V. C., Melo, S. L., \& Oliveira, A. S. (2010). Reconstrução e validação de um Inventário de Socialização Organizacional. Revista de Administração Mackenzie, 11(4), 4-37. doi: 10.1590/S1678-69712010000400002

Carvalho-Freitas, M. N. (2007). A Inserção de pessoas com deficiência em empresas brasileiras - Um estudo sobre as relações entre concepções de deficiência, condições de trabalho e qualidade de vida no trabalho (Tese de doutorado). Recuperado de http:// www.bibliotecadigital.ufmg.br/dspace/bitstream/handle/1843/ CSPO-72UKVU/maria_nivalda.pdf?sequence $=1$

Carvalho-Freitas, M. N., Marques, A. L., \& Scherer, F. L. (2004). Inclusão no mercado de trabalho: um estudo com pessoas portadoras de deficiência. [CD-ROM]. In Associação Nacional de Pós-Graduação e Pesquisa em Administração - ANPAD (Org.), Anais do XXVIII Encontro Anual da Associação Nacional de Pós-Graduação e Pesquisa em Administração, Curitiba, PR.: Autor.

Carvalho-Freitas, M. N., Toledo, I. D., Nepomuceno, M. F., Suzano, J. C. C., \& Almeida, L. A. D. (2010). Socialização Organizacional e pessoas com deficiência. Revista de Administração de Empresas, 50(3), 264-275. Recuperado de http://www.spell.org.br/documentos/ ver/1799/socializacao-organizacional-de-pessoas-com-deficiencia/i/ pt-br

Fishbein, M., \& Ajzen, I. (1975). Belief, attitude, intention and behavior: An introduction to theory and research. Reading, MA: Addison-Wesley.

Goffman, E. (2008). Estigma. Rio de Janeiro: LTC.

Griffin, A. E. C., Colella, A., \& Goparaju, S. (2000). Newcomer and organizational socialization tactics: An interactionist perspective. Human Resource Management Review, 10(4), 453-474. doi: 10.1016/ S1053-4822(00)00036-X

Guedes, L. C. (2007). Barreiras atitudinais nas instituições de ensino superior: questão de educação e empregabilidade (Dissertação de mestrado). Recuperado de https://repositorio.ufpe.br/ handle/123456789/4563

Hair, J. F. Jr., Anderson, R. E., Tatham, R. L., \& Black, W. C. (2005). Análise multivariada de dados ( $5^{\mathrm{a}}$ ed.). Porto Alegre: Bookman.

Hitt, M. A, Miller, C. C., \& Colella, A. (2007). Comportamento organizacional: uma abordagem estratégica. Rio de Janeiro: LTC.

Hulley, S. B., Cummings, S. R., Browner, W. S., Grady, D., Hearst, N., \& Newman, T. B. (2003). Delineando a pesquisa clínica: uma abordagem epidemiológica ( $2^{\mathrm{a}}$ ed.). Porto Alegre: Artmed.
Instituto Brasileiro de Geografia e Estatística. (2010). Censo 2010 Recuperado de http://www.sidra.ibge.gov.br

Lei n. 13.146, de 6 de julho de 2015. (2015, 6 de julho). Institui a Lei Brasileira de Inclusão da Pessoa com Deficiência (Estatuto da Pessoa com Deficiência). Brasília, DF. Recuperado de http://www2. camara.leg.br/legin/fed/lei/2015/lei-13146-6-julho-2015-781174publicacaooriginal-147468-pl.html

Lei n. 8.213 de 24 de julho de 1991. (1991, 24 de julho). Dispõe sobre os Planos de Benefícios da Previdência Social, e dá outras providências. Brasília, DF. Recuperado de http://www.planalto.gov.br/ccivil_03/ leis/l8213cons.htm

Leite, P. V., \& Lorentz, C. N. (2011). Inclusão de pessoas com Síndrome de Down no mercado de trabalho. Inclusão Social, 5(1), 114-129. Recuperado de http://revista.ibict.br/inclusao/article/view/1672

Maccali, N.; Kuabara, P. S.; Takahashi, A. R.; Roglio, K.; \& Boehs, S. T. (2015). As práticas de recursos humanos para a gestão da diversidade: a inclusão de deficientes intelectuais em uma federação pública. Revista de Administração Mackenzie, 16(2), 157-187. doi: 10.1590/1678-69712015/administracao.v16n2p157-187

Ministério do Trabalho e Emprego. (2014). Características do emprego formal segundo a Relação Anual de Informações Sociais. Recuperado de http://portalfat.mte.gov.br/wp-content/uploads/2016/03/ Caracter\%C3\%ADsticas-do-Emprego-Formal-segundo-aRela\%C3\%A7\%C3\%A3o-Anual-de-Informa\%C3\%A7\%C3\%B5esSociais-2014-31082014.pdf

Moreira, L. B., Capelle, M. C. A., Miranda, A. R. A., \& Onuma, F. M. S (2011). Socialização organizacional de pessoas com deficiência: um estudo no Brasil e nos Estados Unidos. In Associação Nacional de Pós-Graduação e Pesquisa em Administração - ANPAD (Org.), III Encontro de Gestão de Pessoas - EnGPR, João Pessoa, PB: Autor.

Neri, M., Pinto, A., Soares, W., \& Costilha, H. (2003). Retratos da deficiência no Brasil. Rio de Janeiro: FGV/IBRE.

Neves-Silva, P.; Prais, F. G., \& Silveira, A. M. (2015). Inclusão da pessoa com deficiência no mercado de trabalho em Belo Horizonte, Brasil: cenário e perspectiva. Ciência \& Saúde Coletiva, 20(8), 2549-2558. doi: 10.1590/1413-81232015208.17802014

Pasquali, L. (2009). Psicometria: teoria dos testes na psicologia e na educação (3 $3^{\underline{a}}$ ed.). Petrópolis: Vozes.

Roulstone, A. (2012). Disabled people, work and employment: a global perspective. In N. Watson, A. Roulstone, \& C. Thomas (Orgs.), Routledge handbook of disability studies (pp. 211-224). Londres: Routledge.

Sassaki, R. K. (2003). Inclusão Social. I Seminário de Políticas Públicas do Município de Limeira. Limeira, SP.

Sassaki, R. K. (2010). Inclusão: construindo uma sociedade para todos ( $8^{\underline{a}}$ ed.). Rio de Janeiro: WVA.

Serrano, C., \& Brunstein, J. (2007). Relações de trabalho, percepção da deficiência e o desenvolvimento profissional do tetraplégico em uma organização pública: em busca do significado da inclusão. In Associação Nacional de Pós-Graduação e Pesquisa em Administração - ANPAD (Org.), I Encontro de Gestão de Pessoas e Relações de Trabalho -EnGPR. Natal, Brasil: Autor.

Shakespeare, T. (2012). Disability in developing countries. In N. Watson, A. Roulstone, \& C. Thomas (Orgs.), Routledge handbook of disability Studies (pp. 271-284). Londres: Routledge.

Stone-Romero, E. F., Stone, D. L., \& Lukaszewski, K. (2006). The influence of disability on role-taking in organizations. In A. M. Konrad, P. Prasad, 
\& J. K. Pringle (Orgs.), Handbook of workplace diversity (pp. 401 430). Londres: Sage Publications.

Suzano, J. C. C., Carvalho-Freitas, M. N., Tette, R. G., Brighenti, C. R. G., \& Vieira-Silva, M. (2014). A percepção dos gestores acerca do desempenho de trabalhadores com diferentes tipos de deficiência. Interação em Psicologia, 18(3), 239-250. doi: 10.5380/psi.v18i3.35498

Van Maanen, J., \& Schein, E. H. (1979). Toward a theory of organizational socialization. Research in Organizational Behavior, 1, 209-264. Recuperado de http://hdl.handle.net/1721.1/1934
Tabachnick, B. G., \& Fidell, L. S. (2007). Using multivariate statistics (5 ed.). Boston: Pearson Education.

Tanaka, E. D. O.; \& Manzini, J. E. (2005). O que os empregadores pensam sobre o trabalho da pessoa com deficiência? Revista Brasileira de Educação Especial, 11(2), 273-294. doi: 10.1590/ S1413-65382005000200008

Wagner, J., III; \& Hollenbeck, J. (2006). Comportamento organizacional (C. K. Moreira, Trad.). São Paulo: Saraiva.

Wood, Jr., \& Caldas M. P. (2007). Comportamento organizacional: uma perspectiva brasileira ( $2^{\mathrm{a}}$ ed.). São Paulo: Atlas.

Gleice Noronha Dias, Mestre em Psicologia pela Universidade Federal de São João del Rei UFSJ, é Psicóloga da Prefeitura Municipal de São João del Rei (PMSJDR). Endereço para correspondência: Rua Aristides Teodoro Ribeiro, $\mathrm{n}^{\circ} 16$ - Recreio das Alterosas - São João del Rei - MG. CEP: 36302-015. Telefone:

(32) 3379-5177. E-mail: gleicenoronha@mgconecta.com.br

Maria Nivalda de Carvalho-Freitas, Doutora em Administração pela Universidade Federal de Minas Gerais (UFMG) e Pós-doutorado em Psicologia pela University of Greenwich (Inglaterra), é Professora Associada do Departamento de Psicologia da Universidade Federal de São João del Rei (UFSJ). E-mail: nivalda@ufsj.edu.br

Raissa Pedrosa Gomes Tette, Mestre em Psicologia pela Universidade Federal de São João del Rei (UFSJ), é Bolsista de Apoio Técnico da Universidade Federal de São João del Rei (UFSJ).

E-mail: raissagomestette@gmail.com 